\title{
UNE ENQUÊTE SUR LE MARCHÉ DU MIEL A PARIS PENDANT L'HIVER 1957-58
}

\author{
PAR
}

\section{R. BORNECK,}

Syndicat des Producteurs de miel de France

et

\section{J. LOUIS et J. LOUVEAUX}

Station de Recherches apicoles, Bures-sur-Yvette (S.-et-O.).

\section{I. - INTRODUCTION}

Je miel compte parmi les plus anciens aliments de l'humanité. Il fut consommé par les hommes bien avant le pain. Il est, sans doute, à l'origine des premières boissons fermentées. Après une éclipse momentanée due à l'introduction du sucre de betteraves au XIXe siècle, il connaît de nos jours une vogue nouvelle. I a mévente du miel est pratiquement inconnue en Firance depuis la dernière guerre ; chaque année des importations viennent combler un déficit de production assez important malgré le développement de l'apiculture.

Pourquoi la consommation du miel est-elle croissante? Il y a sans doute à cela des raisons multiples. Une élévation générale du niveau de vie permet à un plus grand nombre de consommateurs d'acheter un produit alimentaire que son prix permettrait presque de ranger parmi les produits de luxe. La diététique a fait connaitre au grand public par d'innombrables publications les bienfaits d'une alimentation variée ne comportant que des produits sains et naturels. Des campagnes publicitaires en faveur de la gelée royale, du pollen et du miel ont attiré l'attention des masses sur les produits du rucher.

Notre production nationale est-elle en mesure de satisfaire vraiment cette clientèle toujours plus nombreuse? Produisons-nous de bons miels? Nos techniques de préparation et de conditionnement sontelles satisfaisantes? Dans quelle mesure les indications portées sur les emballages sont-elles conformes à la réalité? Nos prix sont-ils homogènes et reflètent-ils vraiment des différences de qualité? I^e commerçant de détail connait-il le miel et sait-il le présenter à sa clientèle? Que désire 
cette clientèle? Autant de questions auxquelles il serait important de pouvoir répondre avec précision si l'on ne veut pas que les campagnes publicitaires entreprises en faveur de la consommation du miel manquent leur but.

I e Syndicat des producteurs de miel de lirance a parfaitement compris l'importance d'une connaissance exacte du marché du miel au stade du détail et, en collaboration étroite avec la Station de recherches apicoles de Bures-sur-Yvette qui mettait à sa disposition les ressources de son personnel et de son matériel scientifiques, il a organisé pendant l'hiver r $957-5^{8}$ une enquête sur la vente du miel à Paris.

Cette enquête a été menée de la façon suivante. Selon notre programme, des enquêteurs bénévoles ( ${ }^{1}$ ), membres du S. P. M. F., devaient effectuer sur l'ensemble de la capitale des achats de pots de miel de $500 \mathrm{~g}$, à raison de 5 échantillons par arrondissement, soit roo échantillons en tout. Pour chaque arrondissement les achats devaient se faire dans des types d'établissements différents : 2 échantillons dans des boutiques peu importantes ou noyennement importantes, 2 échantillons dans les magasins très importants, I échantillon dans un commerce spécialisé en épicerie fine. Par ailleurs, les enquêteurs disposant d'une liste de questions à poser aux commerçants devaient se renseigner de la façon la plus complète possible sur les conditions de la vente. Ces questions étaient en principe les suivantes :

Io Quel genre de miel est-ce?

$2^{\circ}$ D'où vient-il?

$3^{\circ}$ Vient-il directement du producteur?

$4^{\circ} \mathrm{En}$ avez-vous qui soit liquide (ou solide)?

$5^{\circ}$ En avez-vous toute l'année?

$6^{\circ}$ Est-ce toujours le même?

$7^{\circ}$ En vendez-vous beaucoup en ce moment?

$8^{\circ}$ I,e vendez-vous toujours dans le même emballage? Si oui, pourquoi?

$9^{\circ}$ Quel fractionnement $(250,500$, Iooo $\mathrm{g})$ vendez-vous le plus fréquemment?

L,es enquêteurs devaient également relever de façon précise les prix pratiqués pour les différents miels offerts au public et noter l'aspect du commerce ainsi que la présentation du miel dans la boutique.

Rassemblés ensuite à la Station de recherches apicoles de Bures-surYvette les échantillons devaient être soumis à un examen critique très complet : valeur de la présentation, qualités gustatives du produit, spectre pollinique, teneur en eau. Nous reviendrons plus loin sur le détail des méthodes utilisées pour chaque examen. Dans l'ensemble ce programme

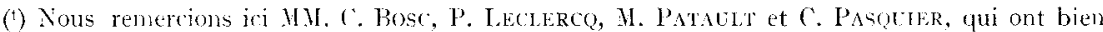
voulu consacrer plusieurs journét's de travail à l'achat daus Paris des échantillons de niel nécesstires à l'enquîte. 
a pu être nis à exécution de façon satisfaisante, compte tenu des difficultés rencontrées. Quatre vingt-dix-huit échantillons ont été recueillis au lieu des roo prévus. On 11'a pas non plus pu obtenir toujours une réponse claire et complète à toutes les questions posées aux commerçants. Mais grâce à la bonne volonté et à l'adresse des enquêteurs, on est arrivé malgré tout à recueillir suffisamment de renseignements pour que les quelques lacunes ne soient pas gênantes. Dans certains cas, par une conversation prolongée avec le commerçant les enquêteurs ont pur an

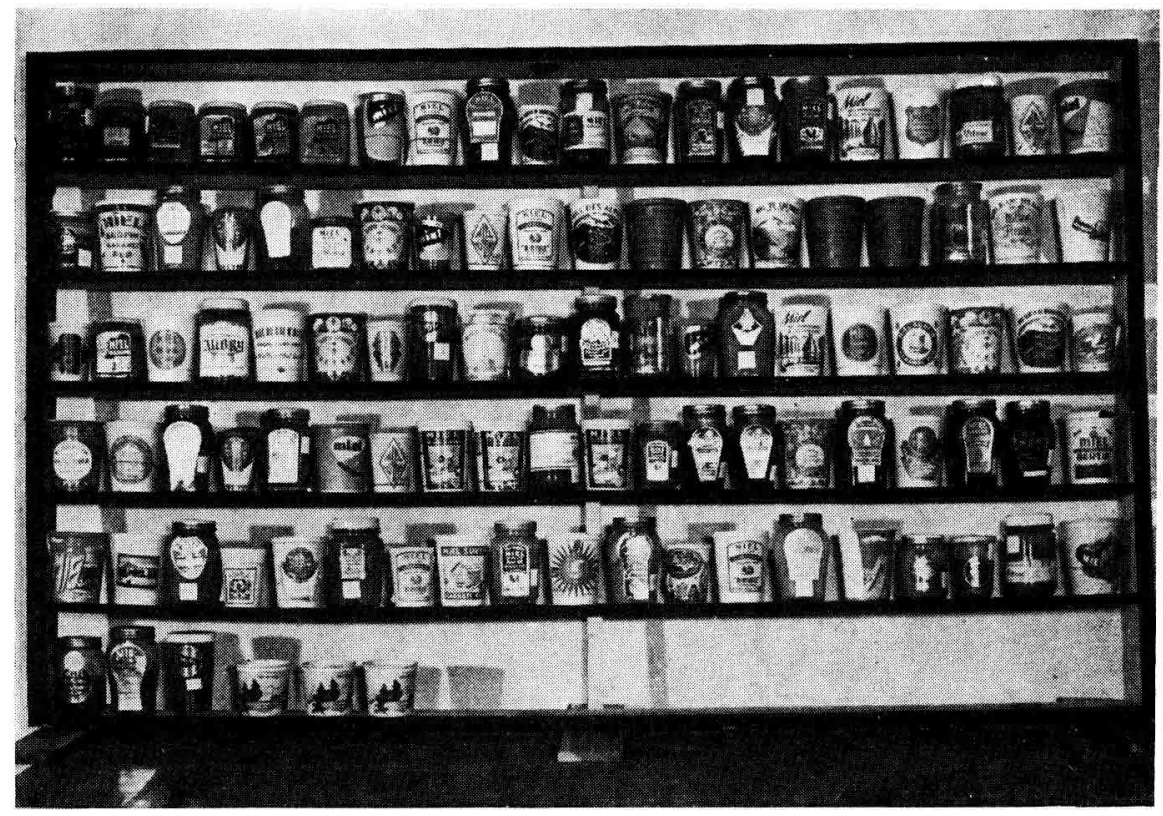

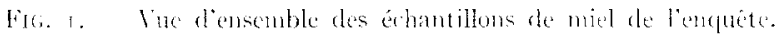

contraire, obtenir des renseignements beancoup plus complets qu'il n'était prévu.

Nons examinerons maintenant les résultats obtenus, tout d'abord sur le plan technique, puis sur le plan commercial.

\section{II. - IRESUSTATS IE L'ENQUETE SUR LE PIAN TECHNTQUE}

Nous avons considéré qu'il était impossible de juger les miels soumis à notre examen au moyen d'une note unique. Nous avons donc procédé à une série de notations portant:

Io Sur la valeur esthétique et les qualités publicitaires de l'emballage indépendamment de sa nature (carton ou verre). 
$2^{\circ}$ Sur la présentation du miel lui-même, abstraction faite de l'anballage; seuls les caractères physiques directement accessibles du miel sont pris en considération.

$3^{0}$ Sur les qualités gustatives du produit; nous nous sommes alors astreints à éliminer nos préférencess personnelles pour ne prendre en considération que le caractère naturel ou non des gonts. Nous arons donc admis a priori que tous les miels naturels et non altérés sont bons.

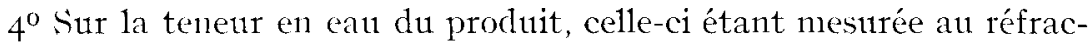
tomètre ${ }^{(1)}$.

$5^{\circ}$ Sur l'exactitude des indications portées sur l'étiquette en distinguant celles qui se rapportent à l'origine géographique, à l'origine florale et à la qualité du miel.

I,es trois premières notes (de o à 5) ont été attribuées par un jury composé de trois apiculteurs professionnels et de trois chercheurs de la Station de recherches apicoles. Ces notes étaient décernées à l'unaminité du jury, l'examen d'un échantillon se poursuivant jusqu'à ce qu'un accord intervienne sur la note paraissant la plus équitable. Ces concessions mutuelles, garantie d'un jugement impartial, ont été fréquenment nécessaires pour concilier des points de vue parfois très divergents, surtout en ce qui concerne la $I^{e}$ et la $3^{\mathrm{e}}$ notation. I a $2^{\mathrm{e}}$ notation n'a donné lieu qu'à des discussions peu importantes, un barême pouvant facilement être établi pour pénaliser les miels physiquement défectueux.

I a $5^{\text {e notation n'a }}$ pu se faire que sur la base d'une analyse pollinique de contrôle. Cette analyse a été faite selon les méthodes classiques qu'il nous parait superflu de rappeler ici. Grâce à cette analyse pollinique nous avons pu établir facilement dans la plupart des cas l'origine géographique et l'origine florale probable des miels. Pour juger de la qualité des miels nous nous sommes reportés aux notes de valeur gustative et de présentation du produit.

Voyons maintenant less résultats obtenus, lesquels ont été regroupés dans le tableau I ${ }^{(2)}$.

\section{'MBIFAL I}

\begin{tabular}{|c|c|c|c|c|c|c|c|}
\hline \multirow{2}{*}{ Notes pour : } & \multicolumn{6}{|c|}{ Vombre des échantillons ityant obtem les notes : } & \multirow{2}{*}{$\begin{array}{c}\text { Tote } \\
\text { moyente }\end{array}$} \\
\hline & o & & 2 & 3 & + & 5 & \\
\hline Io lemballage $\ldots \ldots \ldots \ldots$ & o & 3 & 17 & 22 & . & 26 & 3.5 \\
\hline $2^{\circ}$ la présentation du miel. & 9 & $I_{3}^{3}$ & 25 & 25 & 18 & 8 & 2,5 \\
\hline $3^{\circ}$ les qualités gustatives . . & 6 & .5 & 14 & 18 & 37 & 18 & $\therefore, 25$ \\
\hline
\end{tabular}

(1) Nous remercions II. E. 'l'REBlikT pour le pret de son réfractomètre.

(2) On notera que le nombre des échantillons étamt trís voisin de 100 (08), les chiffrés présentés sont pratiquement assimilables a rles pourcentages. 
I ${ }^{0}$ Note pour l'emballage. - Signalons tout d'abord que sur 98 échantillons, 46 étaient vendus en pots de verre et 52 en pots de carton. Il n'y a donc aucune dominance nette d'un type d'emballage sur l'autre. Le côté commercial de cette question sera examiné plus loin.

Beaucoup d'emballages sont d'une banalité affligeante (note 2). Certains sont franchement laids (note I). Environ la moitié (notes 3 et 4) sont neutres ou agréables. Un quart seulement des pots (note 5) sont réellement esthétiques ou font preuve d'une originalité attirante.

Le pot de verre se prête davantage à une présentation agréable. Le pot de carton semble figé dans une tradition de laideur et de banalité ; il est possible toutefois de faire beaucoup mieux ainsi qu'en témoigne une présentation d'allure très moderne particulièrement réussie (fig. 4 et 5).

Signalons enfin un effort fait par les commerçants de gros pour vendre le miel par $125 \mathrm{~g}$ ou moins dans des verres pouvant être utilisés ultérieurement comme verres à orangeade. Ia multiplicité des types d'emballages utilisés prouve, d'une part que nous ne sommes pas prêts d'atteindre dans ce domaine une standardisation qui paraitrait cependant souhaitable, d'autre part que la question donne lieu à une certaine recherche. Chaque producteur s'efforce de trouver une formule personnelle d'étiquetage et d'emballage. Bien dirigés, ces efforts pourraient conduire à un renouveau du conditionnement du miel qui, dans son ensemble, se présente comme bien vieillot.

Nous noterons pour terminer que, parmi les emballages de verre bon nombre sont dotés d'une fermeture totalement insuffisante, sans aucune étancheité, ou, au contraire trop parfaite, en ce sens que le décapsulage du récipient requiert une force peu commune. Le jury a peiné pendant plus de 5 minutes pour ouvrir un pot de miel à capsule métallique sertie, laquelle n'a consenti à céder que sous les efforts d'une paire de pinces. Ces défauts techniques ont été pris en considération pour l'attribution de la note.

$2^{\circ}$ Note pour la présentation du miel. - On peut dire, sans risque de se tromper beaucoup, que go p. Ioo des miels produits en France sont des miels à cristillisation rapide ou moyennement rapide. En décembre il est normal que presque tous les miels soient cristallisés. Si 1'on établit cependant la proportion des miels solides et des miels liquides au sein des échantillons de l'enquête, on trouve 67 miels solides pour 31 miels liquides. Précisons à ce sujet que nous considérons comme miels solides des miels de première ou de $2^{\mathrm{e}}$ cristallisation entièrement pris en masse. Nous considérons comme miel liquide tout miel parfaitement fluide ou présentant dans sa masse des îlots de cristallisation irréguliers indiquant clairement que le miel a été réchauffé pour être vendu comme miel liquide mais qu'il est incapable de se conserver correctement dans cet état. 


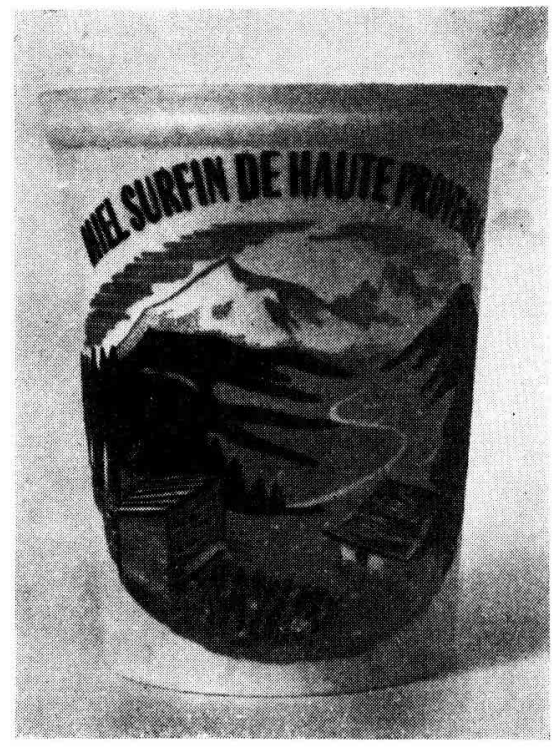

lic. 2 .

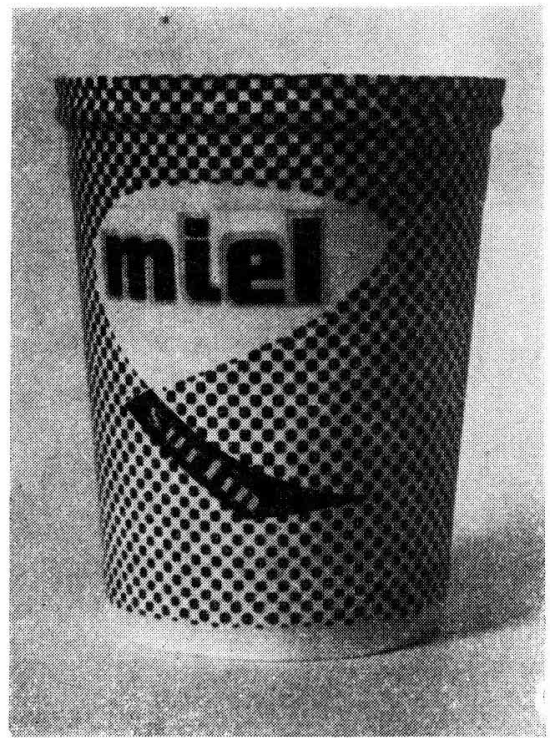

T. IG.

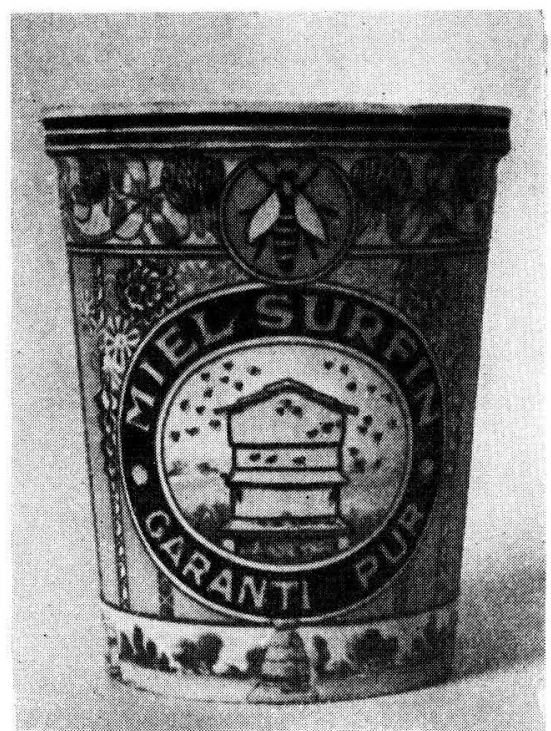

Fis: 3.

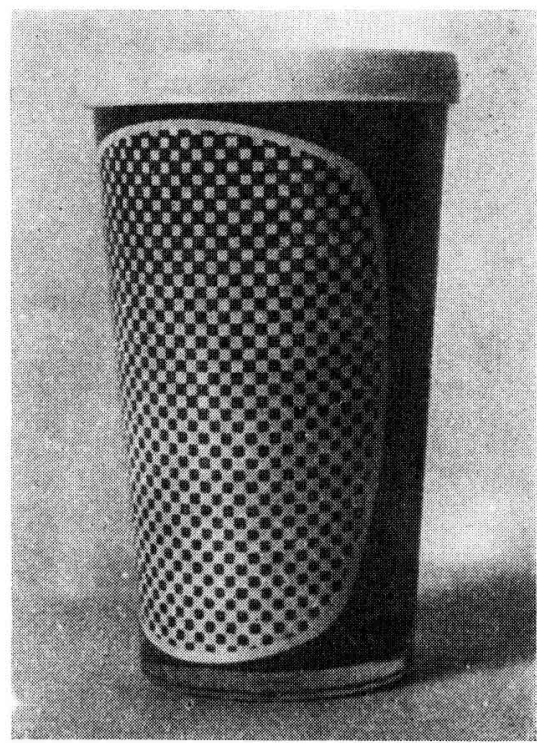

FIt: 5.

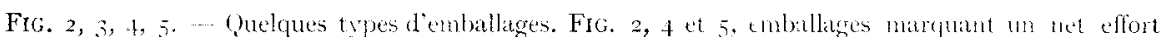
de ${ }^{2}$ résentation. Fa. 3, enballage de conception traditionmelle. 
Deux cas se présentaient pour l'attribution de la note de présentation du miel :

a) Miel en pot de carton. - Dans ce cas la masse du miel n'étant pas directement visible nous avons jugé l'état physique de la surface après ouverture du pot, puis, cette surface étant entamée avec un canif dans le cas de miels solides, l'état physique de la masse. Notre appréciation a porté sur les points suivants :

- propreté et régularité de la surface,

- finesse de la pellicule de glucose en surface,

- finesse de la granulation du miel,

- homogénéité du miel.

Nous avons donc sanctionné les surfaces malpropres, irrégulières, recouvertes d'une pellicule de glucose exagérément épaisse trahissant une remontée d'écume importante, les cristallisations grossières et les états incertains de cristallisation avec ou sans décantation d'une phase solide.

b) Miels en pot de verre. - Dans ce cas l'appréciation était beaucoup plus facile et portait en plus sur les points suivants :

- limpidité pour les miels liquides,

- homogénéité et finesse de la cristallisation pour les miels solides,

- présence de traînées blanches sur les bords du vase.

La moyenne des notes pour la présentation du miel est médiocre puisqu'elle n'est que de 2,5 . Il est frappant de constater que 8 miels seulement ont pu être considérés comme de présentation parfaite (note 5). La moitié des miels ont une présentation défectueuse (notes 2 et 3) par un ou plusieurs points. Un peu moins du quart (notes o et I) se présentent comme des produits très négligés, malpropres, mal cristallisés, donc d'aspect peu engageant. Il n'y a finalement qu'un peu plus du quart des miels (notes 4 et 5 ) qui dénotent chez le producteur ou le négociant une compétence suffisante en matière de présentation des miels. Ceci prouve une insuffisance marquée du matériel d'extraction, d'épuration et de conditionnement du miel.

$\mathrm{I}_{\mathrm{L}}$ vente des miels à l'état liquide est responsable en grande partie des mauvaises notes de présentation. Pour satisfaire une partie de la clientèle qui veut du miel liquide, le conditionneur réchauffe des miels déjà cristallisés sans s'occuper de savoir ce qu'il en adviendra par la suite. Mal refondus, sans contrôle de la température, de la teneur en eau, de la proportion des différents sucres (rapport glucose/lévulose), beaucoup de miels livrés liquides au détaillant se transforment en quelques semaines en un mélange douteux où nagent, au milieu d'un liquide plus ou moins sombre, des cristaux floconneux d'un effet désastreux. Seule, une meilleure connaissance de la technologie du miel, tant chez les producteurs que chez les conditionneurs, permettrait de remédier à cet 
état de choses très nuisible à un développement de la vente du miel. La vente du miel liquide à la clientèle qui le préfère sous cette forme pose des problèmes dont la solution est parfaitement connue des techniciens mais ignorée encore de la masse des producteurs et des négociants.

On peut faire des remarques analogues au sujet de la cristallisation qui constitue, elle aussi, un problème délicat de technologie mais dont la solution est connue. Il est regrettable que les producteurs ignorent encore les moyens d'éviter les traînées blanches sur les parois des récipients ; ces traînées sont dues à la présence de bulles d'air en chapelets qui adhèrent au verre et provoquent la formation de cristallisations de glucose pur, analogues à celles qui constituent la couche blanche de la surface libre. Une mise en pot à température suffisamment élevée (de $1^{\prime}$ 'ordre de $35^{\circ}$ ) n'intervenant que sur un miel bien épuré et déjà très riche en cristaux suffit à éviter tout accident de cristallisation, surtout si on prend soin d'empêcher la formation de bulles d'air au moment où le miel s'écoule dans le récipient.

$3^{\circ}$ Note pour les qualités gustatives du miel. - Comme nous l'avons déjà indiqué plus haut, la note pour les qualités gustatives du miel a été attribuée en faisant abstraction, dans toute la mesure du possible, de l'origine florale. L,es miels à arôme très prononcé, souvent considérés comme de second choix, ne se sont donc pas trouvés automatiquement pénalisés. Nous avons par contre sanctionné tous les mauvais goûts dont l'origine n'est pas à rechercher dans le miel lui-même mais dans les traitements et modifications qu'il a pu subir entre sa récolte et sa mise dans le commerce. C'est ainsi que les miels fermentés, les miels trop riches en pollen, les miels surchauffés, ont reçu, d'une façon générale, des notes très basses ( $\mathrm{O}, \mathrm{I}$ ou 2 selon la gravité du défaut). La note 5 a été réservée à des miels qui, dans leur catégorie (Lavande, Sainfoin, Bruyère, etc...) ont représenté aux yeux du jury le maximum possible de finesse d'arôme, en même temps qu'une concordance parfaite, sur le plan purement gustatif, avec les indications de l'étiquette. Les miels notés 3 on 4 sont de bons miels courants n'ayant pas de vices rédhibitoires mais dont la valeur gustative n'est pas vraiment parfaite.

En fait, si nous n'avons pas sanctionné les miels habituellement considérés comme désagréables au goût (Châtaignier principalement), nous avons quand même baissé la note des miels qui présentent un goût de cette nature dans la mesure où il ne devrait pas se rencontrer dans un miel doté d'une appellation déterminée. Ainsi tout miel du Gâtinais présentant un arrière-goût plus ou moins prononcé de châtaignier s'est trouvé pénalisé alors que nous aurions volontiers accordé la note 5 à un miel pur de Châtaignier qui aurait porté une telle indication sur l'étiquette.

L'examen du tableau I prouve que, en moyenne, et pour l'hiver 


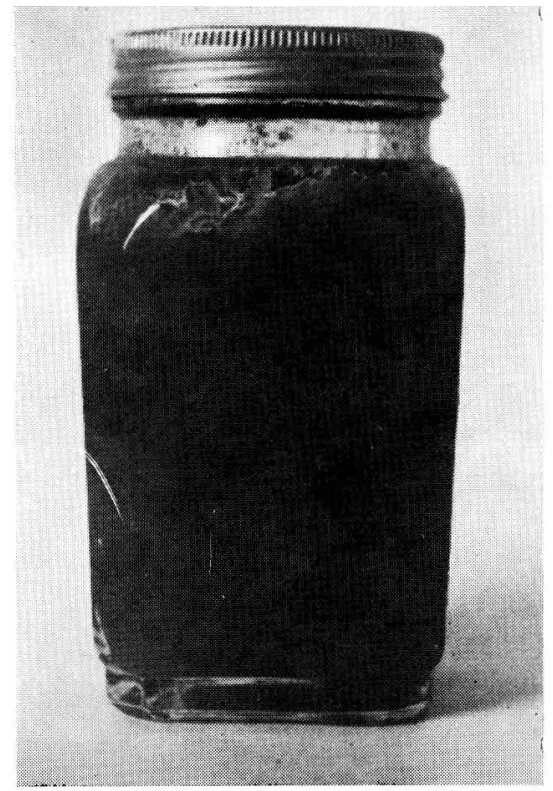

Fig. 6. - Miel liquétié pour lis rente et alian recristallice sous we forme particulierented incsthétique.

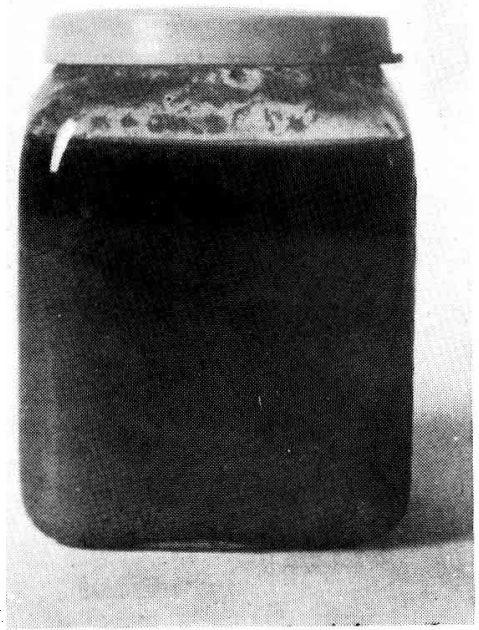

life. 7. Antre défat de cristallisation aver

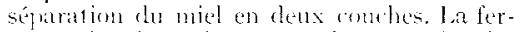
mentation intervent géneralement trés vite dans ces miels.

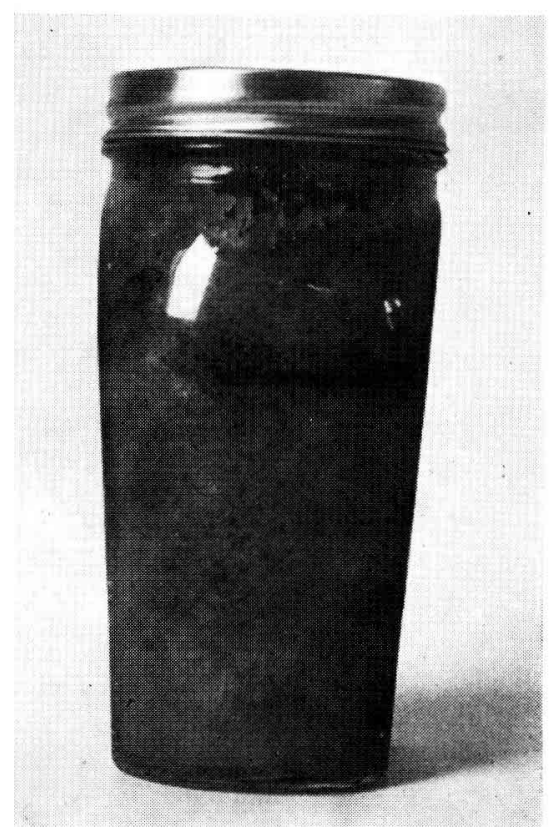

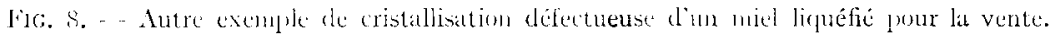




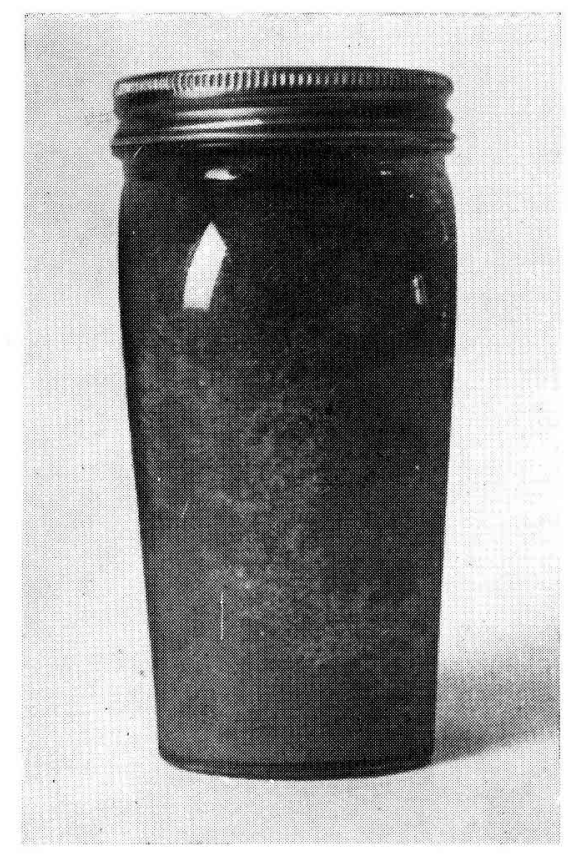

HiG. 9.

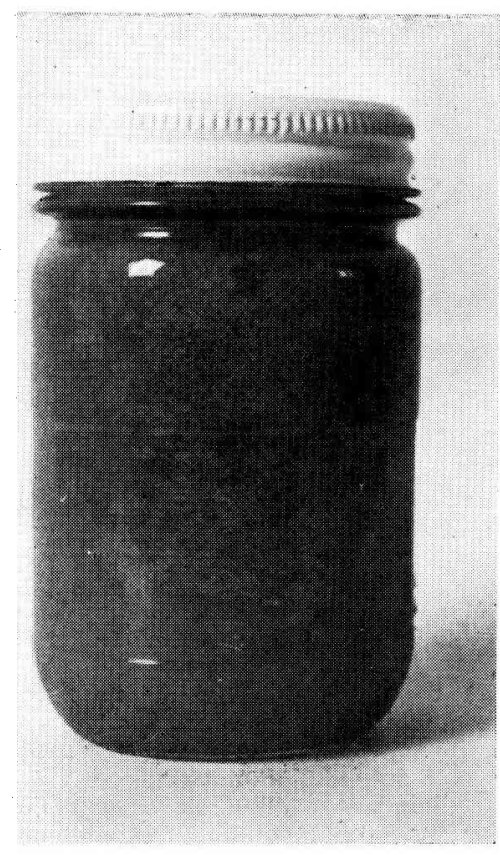

lin. 10 .

FIG. 9 et Io. -- Denx exemples typiques d'incidents de cristallisation interessant la zone de contat miel-verre. Ces accidents sont chus à unc mise en pot à température incorrecte.

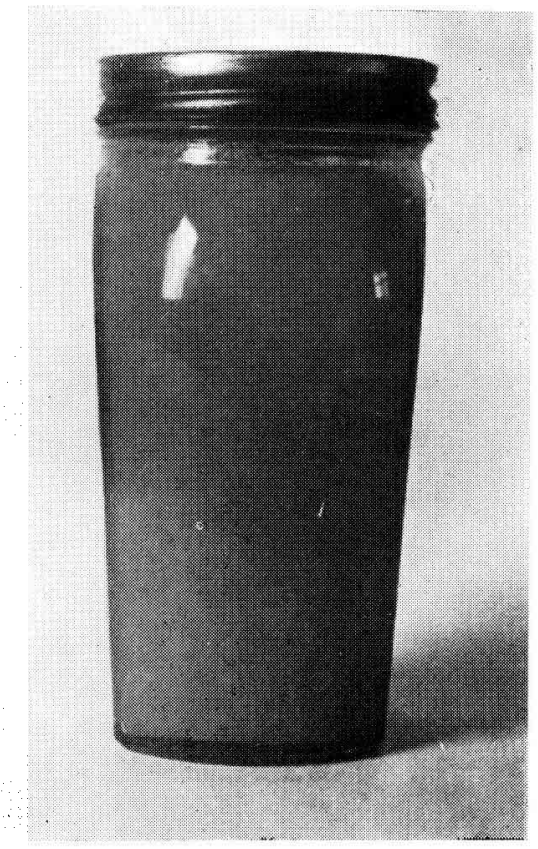

Fig. Ir.-.- Cristallisation parfaite. 


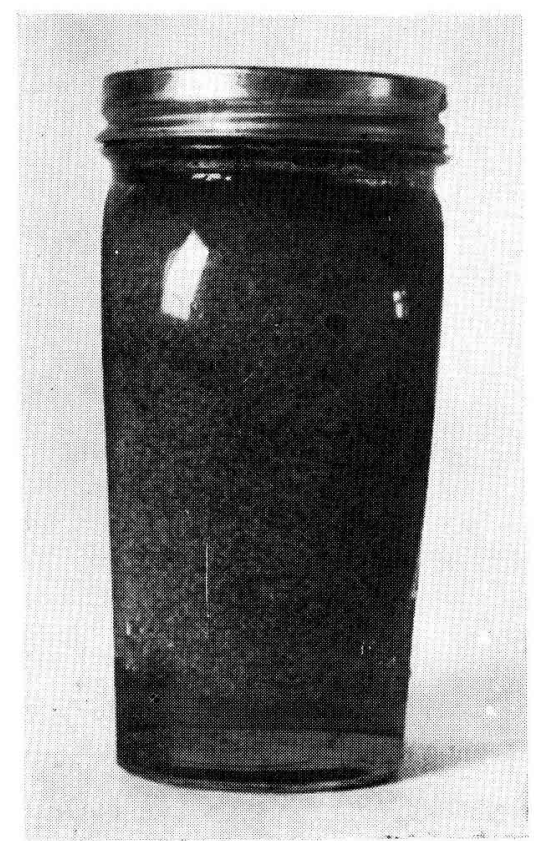

FIG. T2.

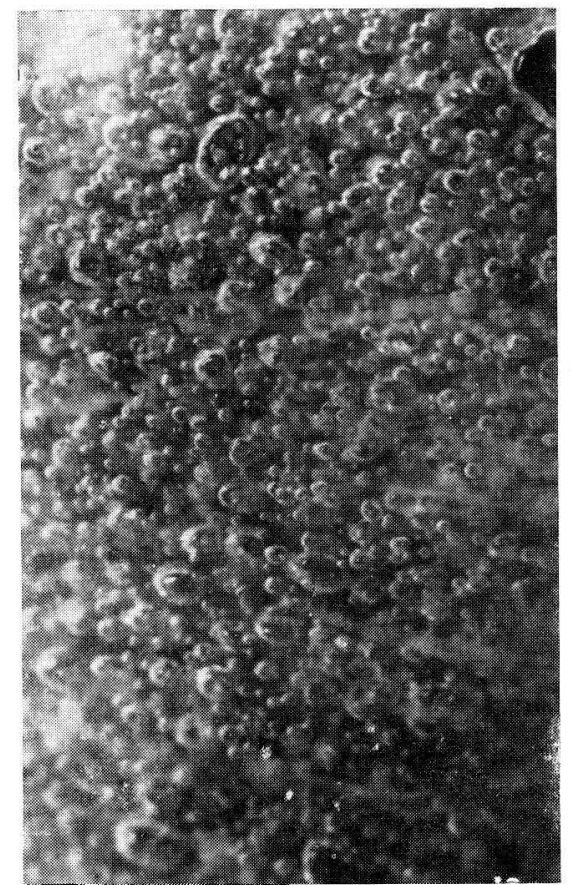

likg. I2 his.

Fro. 12, - Aspect d'un nniel en pleine fermentation. 22 bis : détail des bulles grazeuses dans le miel.

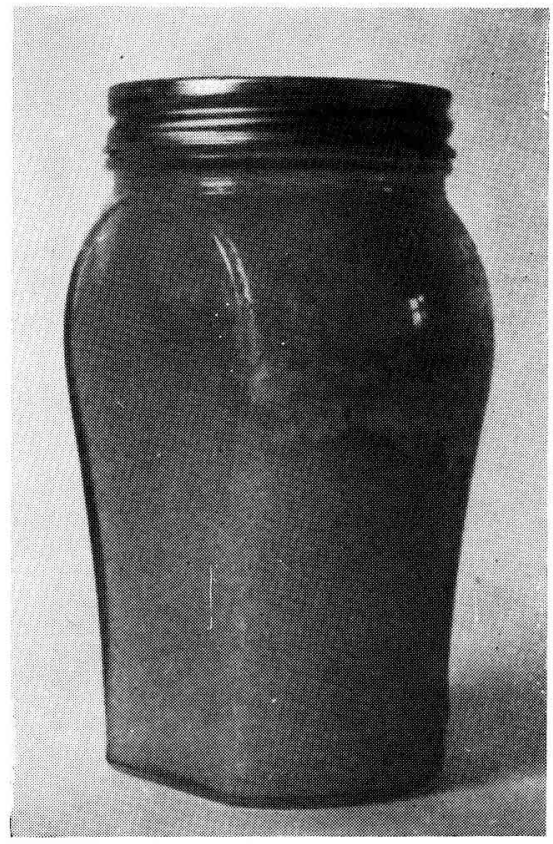

Fig. 1,3.

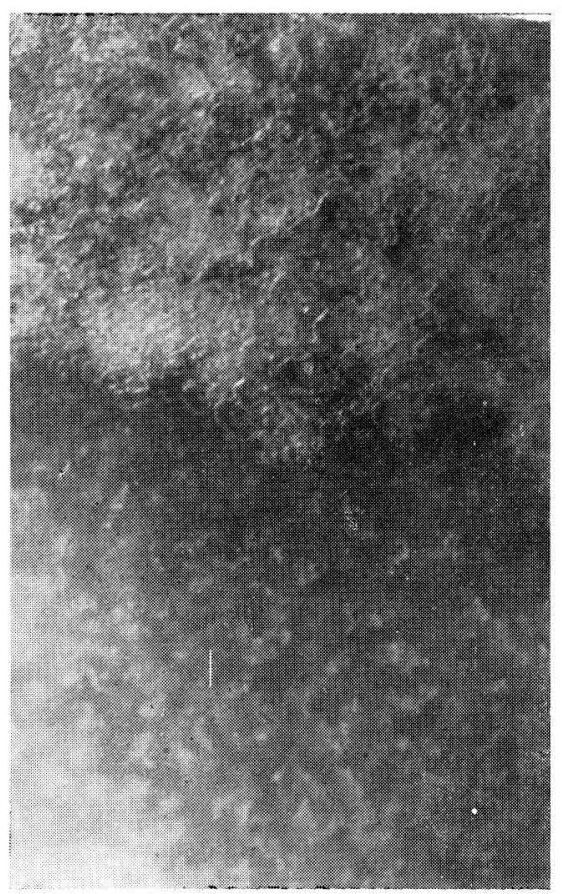

Fig. I3 bis.

Fig. I. - - Aecident de cristallisation ì la surface du micl el défant général de cristallisation daus la mlasse du niel. 13 bis : détail montrant lat présence d'une couche d'air entre micl et verre. 


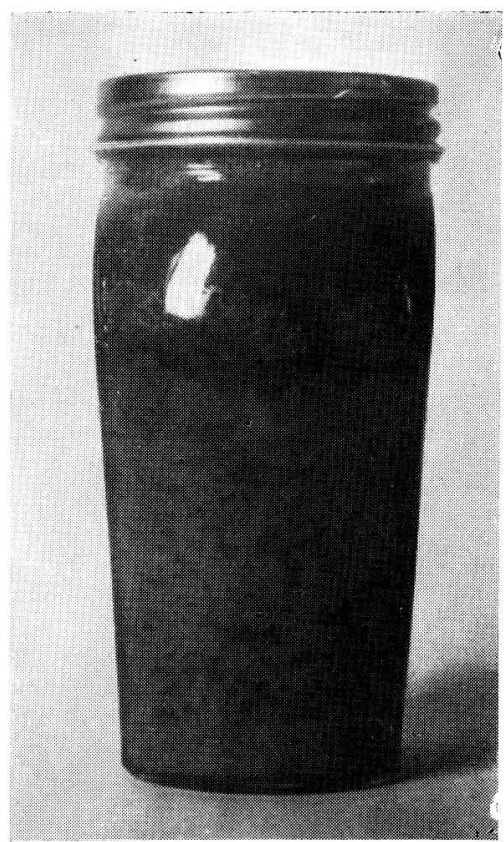

$\mathrm{Fig}, 1+$

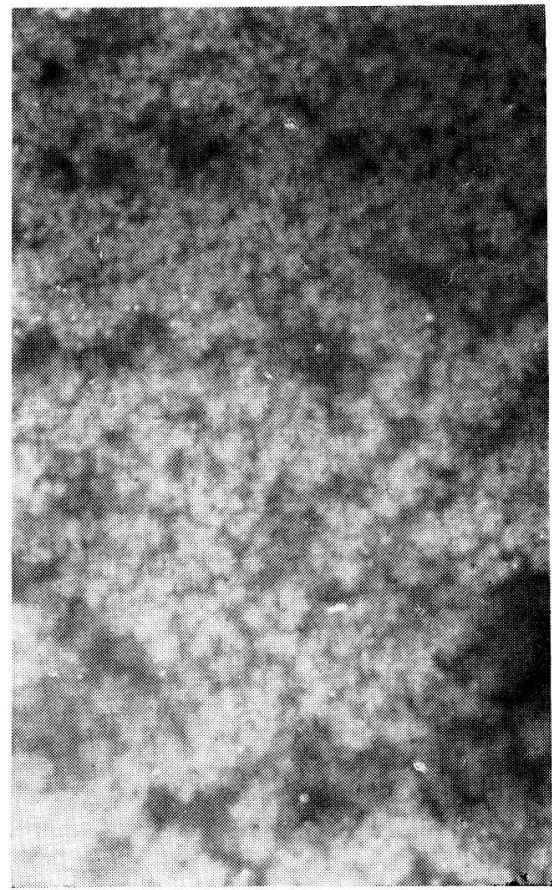

Fig, T..t bis.

Firg. 14 et $\mathrm{I} 4$ bis. - Cristallisation défectueuse et détail des cristaux loconneus dans la masse du miel. 
x $957-5^{8}$, les miels vendus sur le marché parisien sont d'un goût satisfaisant puisque la note moyenne est de 3,25. Il est regrettable qu'un quart des miels soient mauvais ou très mauvais (notes 0 , I et 2); cette proportion est trop élevée au moment oì la propagande s'efforce de développer la consommation; en fait un client sur 4 achète un produit qui ne lui donnera pas satisfaction.

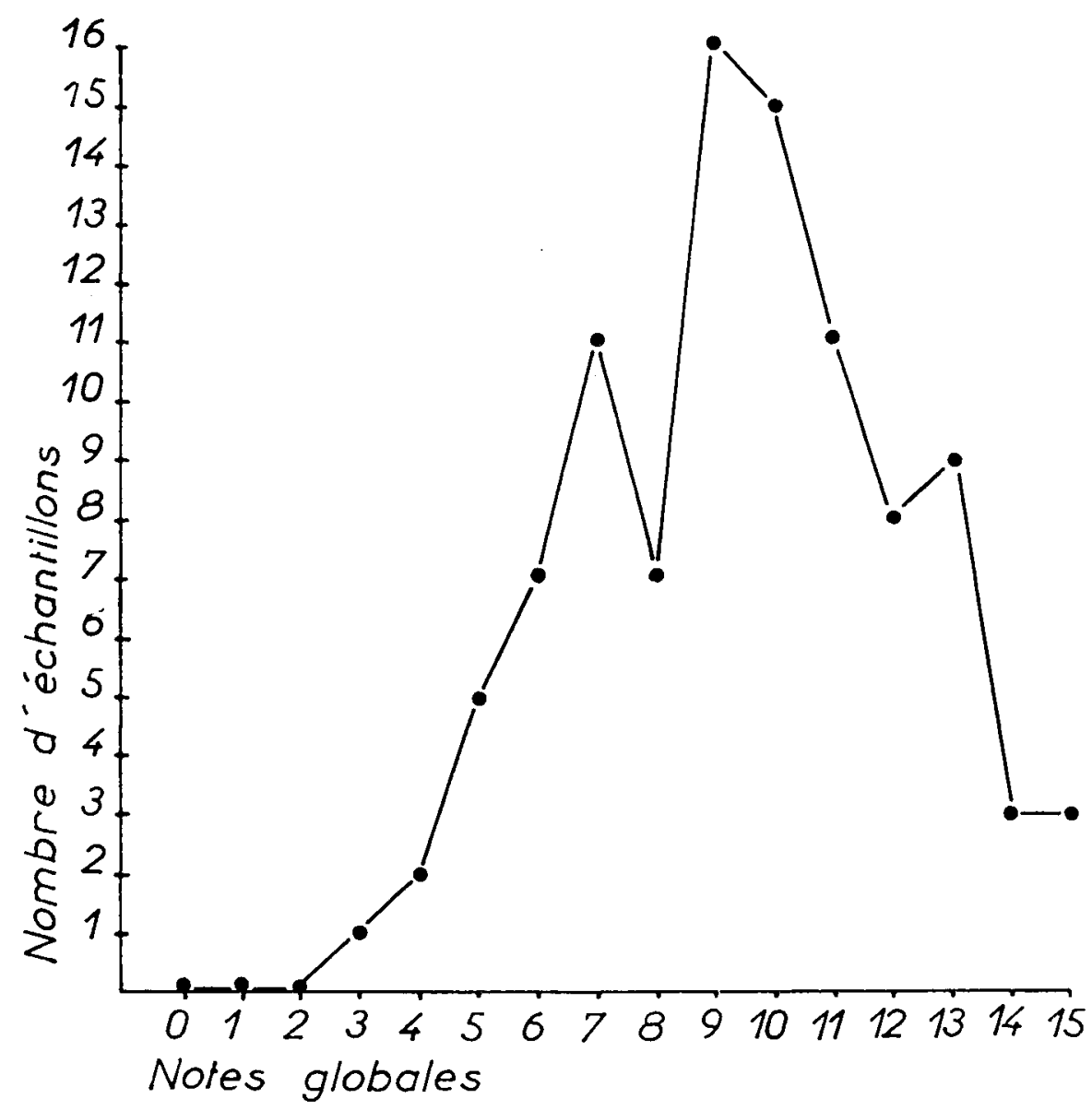

FIG. I5. - Explication dans le texte.

I es $3 / 4$ des miels sont donc bons mais il n'y a pas beaucoup plus d'un miel sur 5 ou 6 qui puisse prétendre à une qualité irréprochable. Certes, il est impossible de ne mettre sur le marché que des miels de tout premier choix ; l'apiculteur récolte ce que les abeilles lui fournissent et il n'est pas maître des caractéristiques gustatives des produits qu'il vend, mais l'élimination des miels ayant un goût vraiment mauvais, parfaitement réalisable par une amélioration générale des techniques de récolte et de conditionnement, suffirait à redresser notablement la situation. 
$4^{\circ}$ Note globale. - Si nous additionnons les trois notes d'emballage, de présentation du miel et de valeur gustative nous obtenons une note globale qui reflète assez bien la qualité du miel puisqu'elle fait intervenir tous les facteurs en fonction desquels sont jugés les produits. La figure ${ }_{5} 5$ nous donne sous forme de graphique la répartition des notes globales. La courbe obtenue présente un sommet principal pour la note 9 sur I5 qui, si elle est au-dessus de la moyenne n'est cependant pas excellente. De grosses possibilités d'amélioration sont révélées par notre graphique.

$5^{\circ}$ La teneur en eau. - L'expérience prouve que tout miel contenant plus de 20 p. Ioo d'eau est un miel susceptible de fermenter facilement. Au-dessus de 20 p. Ioo d'eau la fermentation intervient à peu près fatalement dès que la température est favorable au développement des levures. Ia teneur en eau des miels est donc une donnée fondamentale sous le rapport des facultés de conservation.

La mesure systématique de la teneur en eau des miels soumis à l'enquête nous a révélé que plus de 20 p. Ioo des produits examinés sont trop aqueux. Encore faut-il noter que 1957 fut tune année relativement sèche. En année humide le taux moyen doit s'élever sensiblement. I,e tableau II donne la répartition des pourcentages notés.

'TABLEAU II

Teneur en eau

Inf

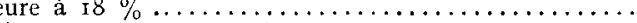

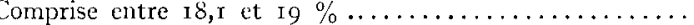

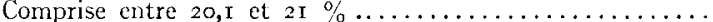

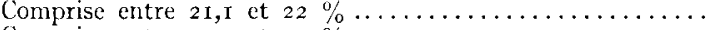

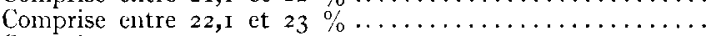

Comprise entre 23 , I et $24 \% \ldots \ldots \ldots \ldots \ldots \ldots \ldots \ldots$

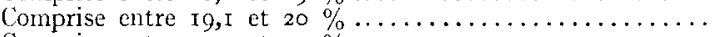

Nombre d'échantillons

Les miels dont la teneur en eau est supérieure à 2 I p. Ioo étaient tous en état de fermentation notoire avec formation d'écume, odeur caractéristique, saveur piquante.

I'apiculture française aurait donc le plus grand intérêt à s'équiper de telle sorte que les miels qui n'ont pas pu être récoltés dans l'état de maturité convenable soient légèrement déshydratés. I'appareillage nécessaire ne représente pas une mise de fonds bien considérable pour les grosses exploitations et il permettrait de rectifier les miels dépassant la limite normale d'hydratation.

$6^{\circ}$ L'exactitude des indications portées sur l'étiquette. - Il convient tout de suite de remarquer (que ces indications sont de plusieurs ordres : géographique (Gâtinais, Hte-Provence, Californie, etc...), botanique (Sainfoin, Trèfle, Acacia, etc...), qualificatif (surfin, extra, etc...), et qu'il est impossible de noter en bloc leur valeur. Nous examinerons 
tout d'abord la correspondance entre l'origine géographique indiquée et le résultat de l'analyse pollinique.

a) Origine géographique. - Sur les 98 échantillons examinés, 33 ne portaient aucune indication d'origine géographique contre 65 qui en étaient pourvus. Pour 37 d'entre eux, soit un peu plus de la moitié, l'indication était indiscutablement vraie ; pour ig elle était notoirement fausse, pour 9 elle était douteuse. Fin ce qui concerne les indications ayant un caractère régional (Gâtinais, Champagne, Hte-Provence, etc...), nous avons fait preuve de beaucoup d'indulgence et considéré comme vrai tout ce qui n'était pas notoirement faux. Il est bien certain que les limites géographiques du Gâtinais ou de la Hte-Provence, en tant que régions naturelles, n'ont pas la précision de limites administratives; tous les géographes ne sont d'ailleurs pas d'accord à leur sujet. Certaines régions voisines peuvent produire des miels suffisamment semblables pour que les sens les plus aiguisés, les méthodes chiniques ou biologiques les plus perfectionnées soient incapables de faire la discrimination. Ces faits suffisent à justifier notre indulgence qui, par contre, ne s'est pas manifestée lorsque la vérité était ouvertement bafouée.

La question des miels d'importation. - Cette question intéresse toujours considérablement les apiculteurs qui s'interrogent sur l'importance des ventes de miels exotiques sous une étiquette nationale ou régionale. Sur 98 miels examinés nous avons trouvé, en nous basant sur les résultats de l'analyse pollinique :

If miels exotiques certains,

I4 mélanges de miels français et de miels exotiques,

$5^{8}$ miels français certains,

Io miels d'origine géographique douteuse.

Sur les I6 miels exotiques certains, 8 portaient une étiquette indiquant leur origine véritable. Les 8 autres ne portaient pas d'indication d'origine géographique ou bien une indication fausse. En ce qui concerne les mélanges de miels français avec des miels exotiques, les proportions des mélanges sont très difficiles, sinon impossible à préciser; elles semblent fort variables. Dans certains cas (généralement classés parmi les douteux) on peut même se demander s'il y a eu mélange à proprement parler ou simplement pollution, les précautions prises pour séparer les catégories de miels n'étant pas suffisantes.

Nous remarquerons encore que tous les miels d'importation ne sont pas forcément des miels de basse qualité, loin de là. La lirance importe aussi bien les très beaux miels d'Acacia de Hongrie ou d'excellents miels de Californie que les miels industriels d'Amérique centrale ou d'Amérique du sud. La fraude sur l'appellation, si elle est moralement condamnable n'est pas toujours préjudiciable à la clientèle; il est moins dangereux pour le prestige du miel de vendre sous le nom de " miel du Gâtinais " 
un beau miel de Trèfle américain dont les caractéristiques sont voisines de celles de nos miels du Gâtinais, qu'un miel récolté sur le châtaignier dans les forêts de 1'Ile-de-France. I a pratique des mélanges n'est pas non plus condamnable dans la mesure où elle peut améliorer les miels et aboutir à un produit meilleur que les produits de départ; il faut toutefois reconnaitre que les mélanges sont faits, le plus souvent, dans le simple but d'abaisser le prix de revient.

b) Origine florale. - L'origine florale des miels n'est indiquée que dans 36 cas sur 98 , soit seulement un peu plus du $\mathrm{x} / 3$. Il faut reconnaître qu'il n'est pas aisé de savoir avec certitude l'origine florale d'un miel et nous approuvons ceux qui renoncent à une indication qui a autant de chances d'être fausse que vraie et qui ne peut être contrôlée, avec une précision toute relative d'ailleurs, que par un examen de laboratoire particulièrement délicat. Sur 36 appellations concernant 1'origine florale I6 sont vraies, I6 sont fausses, 4 sont douteuses. Comment en vouloir d'ailleurs à l'apiculteur qui a commis une erreur d'appréciation sur l'origine florale de son miel? I1 ne possède que des moyens de contrôle souvent fort rudimentaires, sauf dans le cas de grosses miellées d'origine bien connue. Il n'en est pas de même pour certains commerçants peu scrupuleux qui n'hésitent pas, dans un but purement publicitaire, à donner à leurs produits une appellation botanique relevant parfois de la plus haute fantaisie.

c) Origine du miel. - Parmi les 98 échantillons étudiés, 57 portent une indication relative à la qualité du produit. Nous avons relevé un certain nombre de qualificatifs d'intérêt variable tels que "qualité surfine ", "garanti ", " sélectionné ", " surfin ", " extra ", "garanti pur ", "pur ", "naturel ". Ne pouvant entreprendre d'analyser chimiquement tous les miels de l'enquête, ce qui aurait représenté un travail beaucoup trop long, nous ne pouvons pas porter de jugement sur l'exactitude des indication relatives à la pureté ou au caractère naturel des miels. Par contre, nous pouvons fort bien relier la note donnée pour les qualités gustatives à des qualificatifs tels que "surfin ", "extra ", etc...; nous considérons que l'indication de l'étiquette est fausse si le miel dit "surfin " n'a pas reçu au moins la note 4 pour ses qualités gustatives. De même, nous pouvons apprécier grâce à l'analyse pollinique la valeur d'une indication telle que "sélectionné ".

Sur 57 indications relatives à la qualité du miel 3 I sont vraies, I douteuse et 25 sont fausses. On peut donc supposer que les qualificatifs habituellement imprimés sur les emballages, quelle que soit leur destination, ne sont là que par tradition et ne correspondent à rien de précis.

Si nous abordons le problème du point de vue statistique il se présente de la façon suivante : soient Ioo miels dont $50 \mathrm{p}$. Ioo sont surfins et 50 p. Ioo non surfins. Si on applique au hasard l'étiquette surfin sur 
la moitié des pots on doit trouver 25 pots surfins avec l'étiquette "surfin " et 25 pots non surfins avec l'étiquette "surfin ". C'est à très peu de chose près ce que l'on trouve dans la réalité. On peut done considérer que le qualificatif "surfin " a autant de chance d'être vrai que faux, donc, qu'il ne correspond à aucune réalité.

\section{III. - EXAMEN DÉTAILLE DE QUElQUeS CAS PARTICULIERS}

I a publication in-extenso de toutes les fiches qui ont été établies à la suite de notre enquête représenterait un volume beaucoup trop considérable et nous pensons préférable de n'étudier que quelques échantillons représentant des cas typiques aussi bien dans le sens du meilleur que dans le sens du pire. Ceci nous dispensera d'entrer dans le détail des échantillons par trop dépourvus d'intérêt.

Bien entendu nous ne citerons aucun nom de producteur ou de commerçant, chaque niel n'étant désigné que par son numéro d'enregistrement. Afin d'éviter des répétitions nous donnerons les notes par une formule de 6 chiffres correspondant, dans l'ordre, aux notes de o à 5 pour l'emballage, la présentation du miel, les qualités gustatives, puis aux trois notes relatives à l'exactitude des indications de l'étiquette (o = faux, $\mathrm{I}=$ douteux, $2=$ vrai, $-=$ pas d'indication) sur l'origine géographique, l'origine florale, la qualité.

$1^{\text {er }}$ cas. - Miel no $969 / 58$. Notation : $4 / 3 / 2 /-/ / /$. Conditionné par un négociant. Teneur en eau 20,5 p. Ioo. I'analyse pollinique montre qu'il s'agit d'un miel exotique typique en provenance vraisemblable d'Amérique du Sud. La haute teneur en pollen indique un mode d'extraction rudimentaire. L'étiquette indique au consommateur que ce miel contient 6000 gammas de gelée royale et 600 ooo gammas de pollen, soit, pour traduire en clair cette notation pédante, $6 \mathrm{mg}$ de gelée royale et $600 \mathrm{mg}$ de pollen. Le pot de $45^{\circ} \mathrm{g}$ est vendu 370 francs. Étant donné qu'il s'agit d'un miel industriel d'importation, susceptible de fermenter, mal présenté, d'un goût peu agréable, enrichi d'une quantité infime de gelée royale ( 12 à $\mathrm{I} 5 \mathrm{mg}$ par $\mathrm{kg}$ soit 2 à 3 francs au cours actuel), on est en droit de considérer que la vente de ce produit très au-dessus du cours des plus beaux miels constitue un abus de confiance.

2 e cas. - Miel $n^{\circ}$ 974/58. Notation : $2 / 3 / 4 /-/ 0 / 0$. Teneur en eau 20,5 p. Ioo. Conditionné par un négociant. Ce miel est présenté comme miel de Tilleul sélectionné, pur et naturel. Il s'agit d'un miel d'un goût agréable mais très malpropre; il contient en suspension quantité de corps étrangers; la surface est souillée également. L'analyse pollinique montre qu'il s'agit d'un miel français (Sainfoin, Trèfle blanc, Châtaignier, I uzerne, 
Moutarde,...) mais le pollen de Tilleul n'est présent qu'à l'état de traces ; par contre on a identifié quelques pollens typiquement exotiques. Par sa couleur et par son goût ce miel ne rappelle en rien les miels de Tilleul connus. Vendu 330 francs les $500 \mathrm{~g}$ ce miel nous paraît trop cher pour sa qualité ; 1'appellation est totalement fantaisiste.

3e cas. - Miel no 975/58. Notation : 5/4/4/2/-/-/. Teneur en eau 20 p. Ioo. Conditionné par un négociant. L'étiquette porte la mention "Gâtinais ». L'analyse pollinique montre qu'il s'agit d'un beau miel de sainfoin, colza, trèfle blanc, etc... ce qui est en accord avec l'origine annoncée. La présentation du miel est bonne ainsi que le goût ; l'emballage verre marque un effort louable sanctionné par la note 5. Le prix (2 Io francs les $35^{\circ} \mathrm{g}$ ) est honnête pour une marchandise loyale et de bonne qualité. 4 e cas. - Miel no $978 / 58$ : Notation. 5/5/5/2/2/2. Conditionné par un apiculteur récoltant. Teneur en eau I9,5 p. Ioo. Ce miel a obtenu les plus hautes notes dans tous les domaines. Son étiquette ne porte que des indications valables. Il s'agit d'un miel de lavande de Hte-Provence, absolument irréprochable. On regrettera seulement que ce miel soit vendu en pots de carton et non dans des pots de verre le mettant mieux en valeur. Son prix est très modéré : 125 francs les $250 \mathrm{~g}$.

$5^{\mathrm{e}}$ cas.- Miel no $979 / 5^{8}$. Notation : $5 / 5 / 5 /-/ 0 / 2$. Teneur en eau I9 p. Ioo. Conditionné par un négociant. L'emballage verre de ce miel est particulièrement réussi. Seul de son espèce il est vendu entouré de cellophane. Le miel est vendu sous la dénomination "Lavande, surfin, garanti pur ". Le miel est parfaitement liquide et très clair ce qui n'est pas entièrement normal pour un miel de lavande ou de lavandin.L, arôme est typiquement celui du miel de lavandin. L'analyse pollinique révèle un mélange Acacia de Hongrie-Lavandin français. Le prix et de 290 francs les 500 g. Le miel étant parfait à tous les points de vue le mélange opéré ne nuit pas à la clientèle qui, si elle est partiellement trompée sur l'origine, ne l'est pas sur la qualité. L'arôme très prononcé du miel de lavandin supporte fort bien le coupage avec un miel aussi neutre qu'un miel d'Acacia.

$6^{\mathrm{e}}$ cas. - Miel $\mathrm{n}^{\mathrm{o}} 98 \mathrm{o} / 58$. Notation : 5/5/5/2/-/2. Teneur en eau I8,5 p. Ioo. Conditionné par un négociant mais avec indication du producteur. Ce miel des Maures a obtenu les plus fortes notes partout. Son prix est normal : 300 francs les $500 \mathrm{~g}$. Comme pour le $n^{\circ} 978 / 58$ on regrettera seulement la présentation sous carton et non sous verre.

7 e cas. - Miel $n^{0}$ 98I/58. Notation : 4/2/4/2/0/-. Teneur en eau 20 p. Ioo. Miel conditionné par un négociant. Ce miel est intéressant par deux points : ${ }^{0}{ }^{0}$ vendu comme miel liquide il a partiellement recristallisé ce qui justifie sa mauvaise note de présentation. $2^{\circ}$ ) Son appellation est assez curieuse : "Véritable miel des Alpes, garanti naturel, au Cyclamen ». Son spectre pollinique est typiquement provençal ; le nombre 
très élevé des espèces prouve qu'il est un mélange de miels assez variés. par contre il ne nous a pas été possible de trouver de pollen de Cyclamen. On se perd en conjectures sur l'intention du négociant qui a baptisé son miel " au Cyclamen ". S'agit-il d'une marque ou bien veut-on faire croire au client que les abeilles ont récolté ce miel sur des Cyclamens?

8e cas. - Miel no $984 / 58$. Notation : $5 / 2 / 2 / 0 / 0 /-$. Teneur en eau 22 p. Ioo. Miel conditionné par un négociant. Ce miel est en pot de carton de belle apparence portant les indications suivantes : "Miel des Monts d'Auvergile. Tout le parfum et tout le sucre des fleurs des Monts du Forez ". "Absolument pur et naturel ". I, analyse pollinique montre qu'il s'agit en fait d'un miel exotique d'Amérique du Sud (Mimosa pudica très abondante) probablement mélangé à un miel de presse français de basse qualité à base de châtaignier. I,e produit est mauvais, partiellement fermenté, peu appétissant par sa présentation. On comprend aisément qu'un tel produit puisse porter tort à la vente du miel ; son action est d'autant plus néfaste que la présentation de l'emballage est très bonne, donc de nature à allècher le client. Le prix est assez bas : 2 Io francs les $4^{65} \mathrm{~g}$ mais encore trop élevé si l'on considète la qualité du produit vendu.

ge cas. - Miel no $987 / 58$. Notation : $3 / 0 / 0 /-/ /$. 'Teneur en eau 23 p. roo. Conditionné par un négociant. Ce miel est présenté de façon très anodine "Miel de pays ". I'analyse pollinique montre qu'il s'agit d'un miel de presse exotique, soit pur, soit en mélange avec une faible proportion de miel français. L'état de fermentation est très avancé. Le gônt est désastreux autant que la présentation. I,e prix (I 34 francs les $\left.25^{\circ} \mathrm{g}\right)$ est, conme pour le miel précédent, bien trop élevé si l'on tient compte de la qualité déplorable du produit vendu.

10 e cas. - Miel no ror3/68. Notation : $2 / 4 / 1-$ - Teneur en eau 2 I p. Ioo. Miel conditionné par un apiculteur. L'analyse pollinique montre qu'il s'agit d'un miel de sainfoin, trèfle incarnat, trèfle blanc, crucifères. Ce miel pourrait donc être très bon et obtenir partout une note excellente. Mais son emballage carton est laid; le goût du mielest mauvais par suite de fermentations consécutives à l'excès d'humidité. Cet exemple montre comment un produit bon à l'origine, peut finalement échouer en dernière catégorie ou presque par suite d'un manqulue de technicité du producteur.

11 $\mathrm{e}$ cas. - Miel $n^{0}$ I027/58. Notation : 3/1/4/2/-/./. Teneur en eau 20 p. Ioo. Miel conditionné par un apiculteur. L'analyse pollinique montre qu'il s'agit d'un miel de sainfoin parfaitement honnête. I'apiculteur a voulu le vendre liquide. I a recristallisation partielle lui a donné t1n si mauvais aspect que la note de présentation est tombé à I. Nous avons encore là l'exemple d'un miel déprécié par la banalité de son emballage et l'emploi de techniques de liquéfaction défectueuses. 
12e cas. - Miel no I03 $\mathrm{x} / 58$. Notation : 4/2/0/0/-/-/. Teneur en eau I9,5 p. Ioo. Miel conditionné par un négociant. Ce miel est vendu en pot de verre sous une étiquette agréable comme miel du Gâtinais. Très mal cristallisé, sa note de présentation est basse. Son goût est très mauvais. Par ailleurs l'analyse pollinique prouve qu'il s'agit d'un miel exotique presque pur, sale et riche en micro-organismes variés susceptibles de déclencher rapidement la fermentation. Nous avons encore là l'exemple d'un miel susceptible de porter le plus grand tort aux vrais miels du Gâtinais.

13e cas. - Miel $\mathrm{n}^{\mathrm{o}}$ I037/58. Notation : $\mathrm{I} / 5 / 4 / 0 /-/ 0$. Teneur en eau I9, 5 p. Ioo. Miel conditionné par un négociant. Ce produit est présenté comme " aliment spécial pour régimes et cures de diététique; produit en haute montagne ". L'analyse pollinique prouve qu'il s'agit d'un miel exotique fort banal, d'assez bonne qualité mais ne justifiant nullement un panégyrique dont le moins qu'on puisse dire est qu'il est fort exagéré. Le prix ( 320 francs les $500 \mathrm{~g}$ ) est anormalement élevé pour un miel d'importation.

14e cas. - Miel $n^{\circ}$ Io6 $1 / 58$. Notation : 5/3/5/-/2/2. Teneur en eau I8 p. Ioo. Miel conditionné par un négociant. Il s'agit d'un très beau miel de Romarin, origine confirmée par l'analyse pollinique. Ce miel aurait pu avoir la note maximum dans tous les domaines si le conditionneur l'avait vendu liquide ou parfaitement cristallisé. Par suite d'une refonte défectueuse ce miel perd 2 points pour sa présentation.

\section{IV. - RÉSUSTATS DE L'ENQUETE SUR LE PLAN COMMERCTAL.}

Nous avons examiné jusqu'ici divers aspects de l'enquête relevant plus particulièrement du domaine technique. Voyons maintenant les aspects commerciaux des résultats obtenus.

I ${ }^{0}$ Place du miel parmi les autres produits. - Il convient d'insister tout d'abord sur le fait que le miel semble distribué sur l'ensemble du marché parisien d'une manière presque parfaite pour un produit qui n'est pas, après tout, de première nécessité et dont le volume de vente est loin derrière celui d'autres produits sucrés tels que les confitures. En effet, sur 77 points de vente visités dans les 20 arrondissements, un seul ne pouvait offrir de miel. En dépit du caractère assez saisonnier de 1a vente, le produit était offert au client toute 1'année dans 67 magasins, les fournisseurs restant toujours les mêmes. Cependant, dans 5 cas, la vente était uniquement saisonnière et se terminait en fin d'hiver; dans 5 autres cas celle-ci variait selon les livraisons des fournisseurs. Nous pouvons donc affirmer que le miel est bien réparti dans le temps comme dans l'espace sur le marché parisien. 


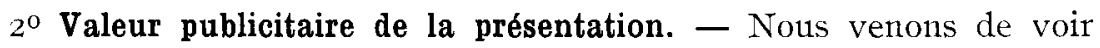
que le miel était correctement distribué dans les magasins d'alimentation mais ceci ne laisse guère préjuger de la manière dont il est offert à la clientèle.

Dans 26 magasins sur $7^{6}$, le miel était à peine visible ou pas visible du tout ; il donnait souvent l'impression d'avoir séjourné dans les rayons durant une période indéterminée. Cet état de choses ne nous a d'ailleurs pas paru lié à une mauvaise gestion de l'affaire.

Dans 39 autres magasins le miel était en bonne ou même très bonne place et le client n'avait pas de peine à le trouver. Il occupait parfois une place assez importante et se présentait sous des formes variées dans les emballages que nous étudierons plus loin.

Enfin, dans 9 magasins nous eûmes la satisfaction de rencontrer une excellente publicité pour le miel. C'était le cas dans les magasins spécialisés mais nous n'avons pas à nous étendre sur ceux-ci qui constituent en eux-mêmes une bonne publicité pour le miel. Notons cependant que l'on pouvait dénombrer dans l'un d'entre eux $I_{7}$ variétés de miels français ou étrangers. Dans d'autres magasins une partie de l'étalage était occupée par une grande quantité de pots de miel (verre ou carton) et le client qui entrait se trouvait alors inconsciemment sollicité par la masse du produit. D'autre part, il pouvait trouver du miel sous des formes très variées, soit en rayons soit en sections ou encore sous forme de mottes de miel à détailler.

I a publicité par affiches semble très rare sur le marché parisien. Nous avons cependant rencontré quelques slogans se rapportant plus spécialement à la gelée royale et dont la présence nous a paru constituer un début de publicité pour tous les produits de la ruche.

D'une manière générale, la vente du miel nous a paru souffrir d'un manque de publicité au stade du marché de détail. De gros progrès restent à faire par les commerçants pour la mise en valeur du miel.

$3^{\circ}$ Emballage et fractionnements. - Pour des raisons diverses, 24 p. Ioo des miels que nous avons achetés avaient une présentation non conforme à la loi. Il nous semble donc utile de rappeler ici certaines des dispositions légales régissant en lirance la vente du miel.

Dénomination. - Le terme " miel "s'applique exclusivement au niel produit par les abeilles. Toutefois, si pendant la période normale de production du miel les abeilles ont été nourries à l'aide de sucre ou de substances sucrées autres que le miel, le produit obtenu ne peut être clésigné que sous la dénomination "miel de sucre".

La dénomination "miel " ne peut être employée pour désigner du miel caramélisé par chauffage ou contenant plus de $25 \mathrm{p}$. Ioo d'eau. I,e qualificatif "pur " ou l'indication d'origine ne peut s'appliquer qu'au miel produit par les abeilles à l'exclusion du miel de sucte (Art. 5 et 7 
du décret du I9/I2/I9Io modifié ou complété par les décrets du I5/7/I92I et $16 / 9 / 1925)$.

Protection du mot "miel ". I e terme " miel " ne peut être employé en France que pour désigner le produit naturel des abeilles. L'expression "miel de fantaisie " ou toute autre appellation similaire est interdite. I es vendeurs de tout produit qui n'aura pas été reconnu pur miel et qui sera vendu sous l'appellation "miel de fantaisie " ou toute autre similaire seront poursuivis conformément à la loi sur les fraudes alimentaires et passibles des peines prévues par ladite loi (Art. I et 2 de la loi du I5/7/ I92I). C'est ainsi que le terme "mielline " par exemple, ne pourra être utilisé à cet effet (Circulaire $\mathrm{n}^{\circ} 5^{6} \mathrm{du} 25 / 7 / \mathrm{Ig} 2 \mathrm{I}$ ). Rentre dans le cas prévu ci-dessus le fait de détenir en vue de la vente, de mettre en vente ou de vendre sous quelque dénomination que ce soit, contenant le mot "miel " ou toute autre expression similaire, des produits ne répondant pas à la définition "miel» (Art. 2 du décret du I6/9/1925).

Miels étrangers. - A partir de leur passage aux frontières et jusques et y compris la mise en vente les miels exotiques devront obligatoirement porter l'indication de leur origine (Art. unique de la loi du 26/3/I93I).

Dans le commerce en gros ou en détail, l'indication du pays d'origine devra figurer sur le couvercle et sur l'étiquette principale des récipients dans lesquels les miels seront offert à l'acheteur. Cette indication sera libellée en caractères latins indélébiles, apparents et bien lisibles de $5 \mathrm{~mm}$ de hauteur minimum.

Miels d'ontre-mer. - Voir la législation très complexe au J. O. du 2/II/I950.

Enfin, nous trouvons en ce qui concerne les modalités d'étiquetage des pots de miel vendus en lirance le décret du I6 novembre I95 I modifiant les dispositions de l'article 28 du I9 décembre I9Io. I 'étiquetage des pots de miel doit comporter les indications suivantes:

a) I a dénomination exacte du produit.

b) L'indication du poids net pour les récipients contenant plus de 250 grammes de miel.

c) I e nom et 1'adresse du fabricant on un numéro spécial de référence de marque. En ce qui concerne cette dernière indication nous croyons devoir préciser que le Service des Iraudes entend par "fabricant " le responsable du conditionnement de la marchandise, nême s'il est apiculteur-producteur.

A. Les emballages. - Ils sont de deux sortes ainsi que nous l'arons signalé plus haut : d'une part les pots de carton paraffiné, d'autre part les verrines spéciales pour le miel. Il est juste de signaler cependant quelques conditionnements dans des verres de fantaisie (verres à boire) ou dans des boites métalliques, ou encore dans des seaux tronconiques de 3 ou $5 \mathrm{~kg}$. Si le nombre des grandes catégories d'emballages est bien 
restreint, il n'en est pas de même des types de fractionnement qui sont, eux, fort nombreux. Toutefois, l'un d'entre eux l'emporte sur les autres.

B. Les fractionnements. - Nous n'avons pas voulu nous borner à l'étude des échantillons effectivement achetés, ce qui aurait pu nous conduire à des conclusions déformées et notus avons interrogé les commerçants pour savoir quel était le fractionnement qu'ils vendaient le plus fréquemment parmi tous ceux qui sont offerts au public. Notre enquête sur ce point particulier a donné les résultats consignés dans le tableau IV établi pour $7^{6}$ points de vente.

TABLEAL IV

\begin{tabular}{|c|c|c|c|}
\hline $\begin{array}{l}\text { Fractionnements les plus demandés } \\
\text { par le public }\end{array}$ & $\begin{array}{c}\text { Nature } \\
\text { de l'emballage }\end{array}$ & $\begin{array}{l}\text { Nombre } \\
\text { de magasins }\end{array}$ & Pourcentasge \\
\hline 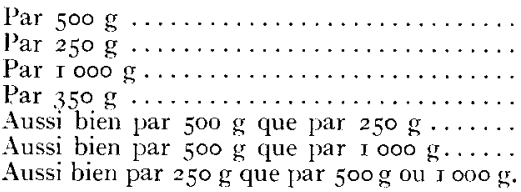 & $\begin{array}{l}\text { Verre ou carton } \\
\text { Verre ou carton } \\
\quad \text { Carton } \\
\quad \text { Verre } \\
\text { Verre ou carton } \\
\text { Verre ou carton } \\
\text { Verre on carton }\end{array}$ & $\begin{array}{r}35 \\
4 \\
3 \\
2 \\
22 \\
9 \\
2\end{array}$ & $\begin{array}{r}45 \% \\
5 \% \\
4 \% \\
3 \% \\
29 \% \\
12 \% \\
3 \%\end{array}$ \\
\hline
\end{tabular}

$\mathrm{X}$. B. Sous la rubrique $500 \mathrm{~g}$ nous avons classé : verres 450 et $500 \mathrm{~g}$ ainsi que cartons $465 \mathrm{~g}$. De même, sous la rubrique $250 \mathrm{~g}$ nous avons classé : verres $250 \mathrm{~g}$ et $350 \mathrm{~g}$, cartons $230 \mathrm{~g}$.

On voit que le pourcentage des ventes en $500 \mathrm{~g}$ (et fractionnements très voisins de $500 \mathrm{~g}$ ) est de loin le plus important. Il n'a pas été possible de discerner s'il était vendu plus de pots de carton que de pots de verre ; un léger avantage semble être du côté du pot de carton du fait de sa moindre valeur d'achat.

Il est certain que le commerce pourrait, s'il le voulait, abandonner tous les fractionnements inférieurs à $500 \mathrm{~g}$ sans diminuer la consommation du miel tout en abaissant notablement ses propres frais de conditionnement.

I es résultats présentés au tableau IV sont confirmés par ceux qui figurent au tableau $V$ relatif aux prix.

$4^{\circ}$ Les prix de détail du miel. - Nous avons opéré ici d'une manière inverse de celle que nous avions retenue pour l'étude des divers fractionnements. En effet, nous nous sommes basés pour le calcul des prix de détail uniquement sur les 98 échantillons dont nous avions fait l'achat, laissant volontairement de côté les renseignements par trop incomplets obtenus au cours de nos conversations avec les commerçants.

Le tableau $V$ (page 24) nous présente le prix payé pour chacun des 98 échantillons achetés au cours de l'enquête. Il s'agit surtout de pots de miel de 450 à $500 \mathrm{~g}$. Nous avons classé les poids nets dans l'ordre croissant, ainsi d'ailleurs que les prix. Les poids sont indiqués nets ou bruts pour nets (b. p. 11.) ; les prix sont indiqués bruts, c'est-à-dire qu'ils 
comprennent le prix de l'emballage et, parfois, des consignes remboursables.

Le prix moyen brut de détail d'un kilo de miel ressort à 625 francs. Ce prix moyen a été obtenu en divisant le montant de nos dépenses (25647 francs) par le total des poids nets (4I kg).

L'échelonnement des prix. - Notons qu'à l'examen du tableau $r$ on retrouve quelques règles commerciales bien connues :

- Le prix du miel augmente en raison inverse du fractionnement.

- Les emballages de verre coûtent légèrement plus cher que les emballages de carton.

$$
\text { TABIEAU V }
$$

Prix des échantillons de miel achetés au cours de l'enquête.

\begin{tabular}{|c|c|c|c|c|c|c|c|c|}
\hline & $\begin{array}{c}\text { Verre } \\
500 \mathrm{~g} \\
\text { net }\end{array}$ & $\begin{array}{l}\text { Carton } \\
465 \mathrm{~g} \\
\text { b.p.n. }\end{array}$ & $\begin{array}{c}\text { Verre } \\
450 \mathrm{~g} \\
\text { net }\end{array}$ & $\begin{array}{c}\text { Verre } \\
45^{\circ} \mathrm{g} \\
\text { net }\end{array}$ & $\begin{array}{c}\text { Verre } \\
350 \mathrm{~g} \\
\text { net }\end{array}$ & $\begin{array}{c}\text { Verre } \\
250 \mathrm{~g} \\
\text { net }\end{array}$ & $\begin{array}{l}\text { Carton } \\
230 \mathrm{~g} \\
\text { b.p.11. }\end{array}$ & $\begin{array}{c}\text { Verre } \\
200 \mathrm{~g} \\
\text { net }\end{array}$ \\
\hline & $\begin{array}{c}\text { Frs } \\
220 \\
250 \\
255 \\
260 \\
285 \\
295 \\
295 \\
300 \\
300 \\
300 \\
310 \\
320 \\
330 \\
330 \\
330 \\
330 \\
355\end{array}$ & $\begin{array}{c}\text { Frs } \\
210 \\
220 \\
220 \\
220 \\
228 \\
230 \\
230 \\
235 \\
240 \\
240 \\
240 \\
240 \\
245 \\
246 \\
260 \\
265 \\
265 \\
270 \\
275 \\
280 \\
285 \\
290 \\
290 \\
290 \\
295 \\
295 \\
295 \\
300 \\
300 \\
300 \\
310 \\
310 \\
310 \\
310 \\
315 \\
315 \\
315 \\
320 \\
325 \\
325 \\
330 \\
335 \\
380\end{array}$ & $\begin{array}{l}\text { Frs } \\
270 \\
270 \\
276 \\
280 \\
290 \\
295 \\
300 \\
304 \\
310 \\
320 \\
325 \\
325 \\
325 \\
330 \\
340 \\
340 \\
340 \\
370\end{array}$ & $\begin{array}{l}\text { Frs } \\
206\end{array}$ & $\begin{array}{l}\text { Frs } \\
203 \\
210 \\
210 \\
220\end{array}$ & $\begin{array}{l}\text { Frs } \\
165 \\
185\end{array}$ & $\begin{array}{c}\text { Frs } \\
124 \\
125 \\
134 \\
140 \\
145 \\
15 \circ \\
150 \\
160 \\
160 \\
175\end{array}$ & $\begin{array}{l}\text { Frs } \\
\text { I15 } \\
\mathrm{I} 25 \\
\mathrm{r} 33 \\
\mathrm{I} 38 \\
\mathrm{I} 40\end{array}$ \\
\hline $\begin{array}{l}\text { Total } \ldots \ldots \ldots \ldots \\
\text { Prix moyen..... }\end{array}$ & $\begin{array}{r}5065 \\
298\end{array}$ & II 459 & $\begin{array}{r}5160 \\
312\end{array}$ & $\begin{array}{l}206 \\
206\end{array}$ & $\begin{array}{l}843 \\
2 \mathrm{II}\end{array}$ & $\begin{array}{l}350 \\
175\end{array}$ & $\begin{array}{r}\mathrm{I} 463 \\
\text { I } 46\end{array}$ & $\begin{array}{l}60 I \\
131\end{array}$ \\
\hline
\end{tabular}


Notons également quelques anomalies assez surprenantes :

- Dans un même emballage, les prix plafonds et les prix planchers sont séparés par une marge très importante dans laquelle s'inscrivent d'ailleurs tous les prix intermédiaires. Cette marge peut atteindre I70 francs. sur $465 \mathrm{~g}$ de miel.

- Il est surprenant de voir le prix moyen des pots de verre de $450 \mathrm{~g}$ supérieur à celui des pots de $500 \mathrm{~g}$.

Nous nous sommes efforcés de rechercher une explication logique à ces différences et notamment nous avons tenté d'établir un certain nombre de rapports entre le prix et divers facteurs pouvant l'influencer.

Rapport prix-origine géographique du miel. -- L'examen de nos fiches ne nous donne sous ce rapport aucun résultat intéressant.

Rapport prix-qualité du conditionneur. - Nous avons noté que 29 p. Ioo des échantillons provenaient directement d'apiculteurs producteurs, le reste passant par les grossistes. Il était permis de supposer que les différences de prix pouvaient avoir leur origine dans la qualité (producteur ou grossiste) du conditionneur. En fait, il ne nous a pas été possible de mettre en évidence une relation de cet ordre.

Rapport prix-qualité du miel. - Il nots restait à examiner 1'hypothèse bien normale d'un rapport étroit entre les prix pratiqués et la qualité de la marchandise offerte. Or, il n'en est pas toujours ainsi; nous avons d'ailleurs déjà noté le fait dans la première partie de ce travail. Ce n'est que dans le cas des miels de lavande du sud-Est que nous avons pu trouver une relation positive prix-qualité du miel.

Nous pensons finalement qu'il faut rechercher dans d'autres directions l'explication des importantes différences de prix entre des produits de même nature. Ceci pourrait faire l'objet d'une étude particulière, sans doute assez longue.

\section{CONCLUSIONS}

I'enquête que nous avons menée pendant l'hiver I957-58 sur le marché parisien du miel nous amène à formuler un certain nombre de remarques que nous pouvons résumer comme suit :

- I es miels présentés à la clientèle sont généralement bons, quelquefois très bons mais on regrettera de trouver encore dans les magasins des produits de basse ou très basse qualité dans une proportion non négligeable (25 p. roo environ). Un effort devrait être fait pour l'élimination progressive et complète du marché de tous les miels de mauvaise qualité susceptibles de détourner la clientèle de la consommation du miel.

- I a mauvaise qualité des miels est due le plus souvent à des techniques de récolte défectueuses ou à des manipulations maladroites. 
Il est regrettable entre autres que la législation permette la mise en vente de miels contenant $25 \mathrm{p}$. Ioo d'eau; cette tolérance est beaucoup trop grande. Le producteur et le négociant ne devraient livrer au commerce de détail que des miels titrant au plus 20 p. Ioo d'humidité. La proportion des miels sales est, elle aussi, trop élevée.

- I,es problèmes de liquéfaction et de cristallisation du miel qui ont pourtant reçu des solutions à peu près définitives continuent à constituer l'écueil majeur auquel se heurtent tant les apiculteurs que les négociants et industriels. On reste frappé du manque de technicité des différentes professions appelées à manipuler le miel.

- La fraude sur les appellations, si elle est assez fréquente, ne revêt cependant pas l'ampleur que l'on aurait pu craindre. Elle semble le fait d'une petite minorité. On pourrait toutefois souhaiter une surveillance plus active de la part des services intéressés.

- A tous les stades de la commercialisation on peut constater que de gros efforts restent à faire pour améliorer la présentation des produits. On trouve encore trop d'emballages inesthétiques ou ne répondant pas aux exigences de la publicité moderne.

- Une certaine standardisation est souhaitable en ce qui concerne la contenance des divers récipients. L,e nombre des modèles de pots de carton et de verre est inutilement élevé.

- Il parait enfin souhaitable de voir intervenir une certaine homogénéisation des prix. I eur dispersion semble très exagérée et n'est pas en rapport avec la qualité des différents produits mis en vente.

Nous conclurons finalement sur la nécessité pour la production et le commerce de sortir hardiment de la routine et d'utiliser largement les techniques modernes de traitement, de conditionnement et de vente du miel. C'est à cette seule condition que les efforts publicitaires actuellement réalisés trouveront leur pleine efficacité.

\section{SUMMARY}

During I957-1958 winter, french syndicate of honey producers, working with honeybee research station of Bures-sur-Yvette, made an inquiry on the sale of honey in Paris. About one hundred honey jars, coming from all parts of the town, were bought. Quality, presentation, water content were carefully examinated, exactitude of indications written ou labels were controlled by pollen analysis. It result from this inquiry that honeys presented for sale, are generally good, but also, that about $25 \%$ from these honeys are really of a low quality.

Low-quality honey's causes are generally : faulty removing or clumsy handling. We are sorry that actual legislation allows honeys containing more than $20 \%$ of water, to be put on sale.

Concerning honey presentation, liquifaction and cristallization are the most important difficulties for beekeepers and merchants. 
Fraudulent indications on labels, seem to be the fact of a minority of course we must remind this, but on that very point we could fear to find the things worse. Big efforts must still be made to improve and unify presentation. It seems that number of packing models is uselessly high.

Prices are very differents these differences cannot be explain sometimes.

The effort of advertising now done to favour the use of honey, should be accompanied by a general improvement of quality and presentation.

\section{ZUSAMME,NFASSUNG}

Während des Winters I $957 / 58$ hat das Syndikat der französischen Honigproduzenten, in Zusammenarbeit mit der Station de Recherches Apicoles in Bures-sur-Yvette, eine Untersuchung über den Honighandel in Paris unternommen. Etwa Ioo Honigproben wurden im ganzen Gebiet der Stadt Paris gekauft und einer eingehenden Untersuchung unterworfen, in Bezug auf Qualität, Verpackung, Wassergehalt und Richtigkeit der in der Etikette enthaltenen Angaben; letzteres geprüft mit Hilfe der Pollenanalyse. Diese Untersuchung zeigte, dass die im Handel dargebotenen Honige im allgemeinen gut sind, dass jedoch etwa $25 \%$ davon eine mindere Qualität aufweisen.

Die schlechte Qualität dieser Honige ist zum guten Teil auf fehlerhafte Gewinnungsmethoden oder ungeeignete Behandlung zurïckzuführen. Es ist bedauerlich, dass die heutige Gesetzgebung den Verkauf von Honigen, mit einem iiber $20 \%$ liegenden Wassergehalt duldet.

Die Probleme der Verfliissigung and Kristallisation des dargebotenen Honigs bilden die gefährlichste Klippe, an welcher Bienenzüchter, wie Honighändler heute noch scheitern.

Falsche Deklarationen scheinen einen geringeren Anteil zu bilden als zu befürchten war.

Es wäre erstrebenswert, die Darbietung von Honig im Handel zu verbessern und zu vereinheitlichen. Vor allem sind unnötig viele Typen der Verpackung vorhanden. Der Preis zeigt eine sehr grosse Streuung, deren Ursache nicht abgeklärt werden konnte.

Die gegenwärtig unternommenen Anstrengungen zur Steigerung des Honigkonsums sollten mit einer allgemeinen Verbesserung der Qualität und der Verpackung der im Handel dargebotenen Produkte verbunden werden. 